\title{
Editorial
}

\section{Theory and Applications of Complex Networks}

\author{
Tingwen Huang, ${ }^{1}$ Zhichun Yang, ${ }^{2}$ and Chuandong $\mathrm{Li}^{3}$ \\ ${ }^{1}$ Texas A\&M University at Qatar, P.O. Box 23874, Doha, Qatar \\ ${ }^{2}$ School of Mathematics and Statistics, Chongqing Normal University, Chongqing 400047, China \\ ${ }^{3}$ School of Information, Southwest University, Chongqing 400715, China
}

Correspondence should be addressed to Tingwen Huang; tingwen.huang@qatar.tamu.edu

Received 30 October 2013; Accepted 30 October 2013; Published 20 January 2014

Copyright (C) 2014 Tingwen Huang et al. This is an open access article distributed under the Creative Commons Attribution License, which permits unrestricted use, distribution, and reproduction in any medium, provided the original work is properly cited.

Between two randomly selected persons in the world, roughly how many friends are there connecting them together? How are people infected by epidemics such as AIDS and bird flu all over the world? All of these are typical examples of complex networks. The impact of complex and dynamical networks pervades the physical, biological, and social sciences. Its influence on modern engineering and technology is notable and will be far-reaching. Since the discoveries of Watts and Strogatz on small-world networks, and those of Barabási and Albert on scale-free networks, many studies on various realworld networks have been carried out and reported from different points of view. The recent study of complex networks has attracted interest to the modeling and understanding of complex networks. In the past few decades, we have witnessed great success of the study in the coordination dynamics or cooperative collective behaviors of autonomous agents in complex networks.

This special issue focuses on modeling, analysis, control, and applications of real-world complex systems.

The paper entitled "Photovoltaic power prediction based on scene simulation knowledge mining and adaptive neural network" analyzes influence of light intensity, day type, temperature, and season on photovoltaic power.

The paper entitled "Using software dependency to bug prediction" uses several quantitative network metrics to explore their relationships with bug prediction in terms of software dependency.

The paper entitled "ACO-based routing algorithm for cognitive radio networks" proposes an ACO-based on-demand routing algorithm to tackle the routing problem.

The paper entitled "Modeling computer virus and its dynamics" presents a novel computer virus model.
The paper entitled "An evaluative study on the choice of regional strategic emerging industry based on dematel: heilongjiang province as an example" expands the DEMATEL method to the field of the complex weighted networks.

The paper entitled "Impulsive consensus for leaderfollowing multiagent systems with fixed and switching topology" establishes the consensus problem of the leaderfollowing multiagent system.

The paper entitled "User demand aware grid scheduling model with hierarchical load balancing" proposes the algorithm of hierarchical load balancing approach with user demand aware grid scheduling.

The paper entitled "A resilience approach to symbiosis networks of ecoindustrial parks based on cascading failure model" constructs a cascading model with tunable parameters for symbiosis networks of EIPs.

The paper entitled "Persistent homology of collaboration networks" analyzes the structure of weighted networks using persistent homology.

The paper entitled "Memristive chebyshev neural network and its applications in function approximation" presents a novel Chebyshev neural network combined with memristors to perform the function approximation.

The paper entitled "A new multi-objective evolutionary algorithm for community detection in dynamic complex networks" develops a novel multiobjective algorithm for detecting communities in dynamic networks.

The paper entitled "Memristive perceptron for combinational logic classification" proposes a new synaptic weight update rule.

The paper entitled "Resonant tunneling diodes-based cellular nonlinear networks with fault tolerance analysis" presents a 
resonant tunneling diodes cellular neural/nonlinear network (RTD-CNN) model.

The paper entitled "Observer-based feedback stabilization of networked control systems with random packet dropouts" designs an adaptive controller of Networked Control Systems with Random Packet Dropouts.

\section{Acknowledgments}

We would like to express our sincere gratitude to the reviewers whose professional comments and suggestions guaranteed the high quality of these selected papers. Also, we are grateful to the authors for their contributions. This work is partially supported by the National Natural Science Foundation of China under Grant no. 10971240 and Grant no. 61374078. This paper was also made possible by NPRP Grant no. NPRP 4-1162-1-181 from the Qatar National Research Fund (a Member of Qatar Foundation). The statements made herein are solely the responsibility of the authors.

Tingwen Huang Zhichun Yang Chuandong Li 


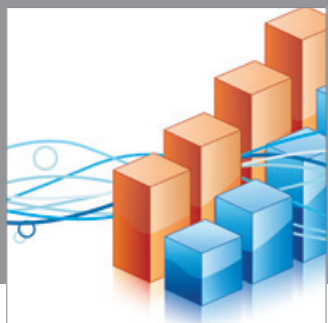

Advances in

Operations Research

mansans

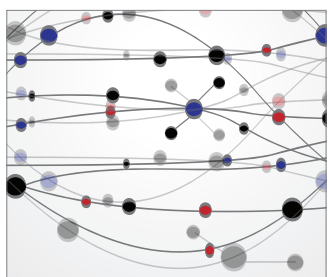

The Scientific World Journal
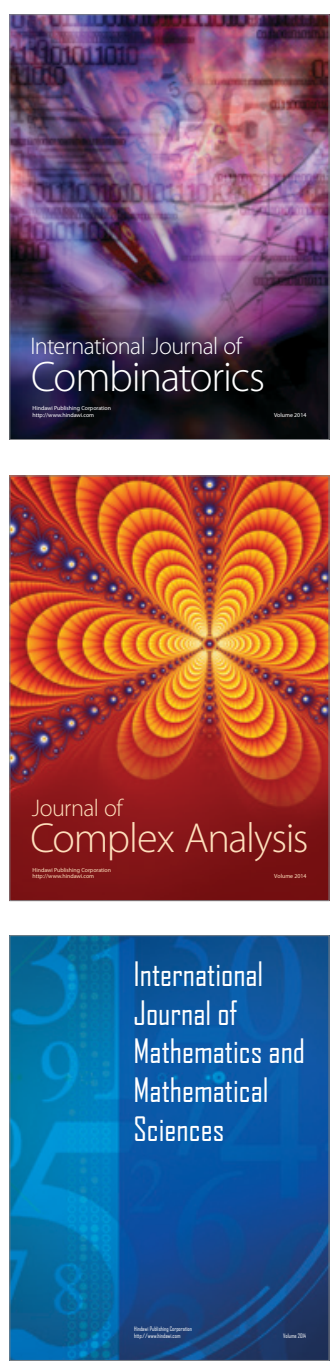
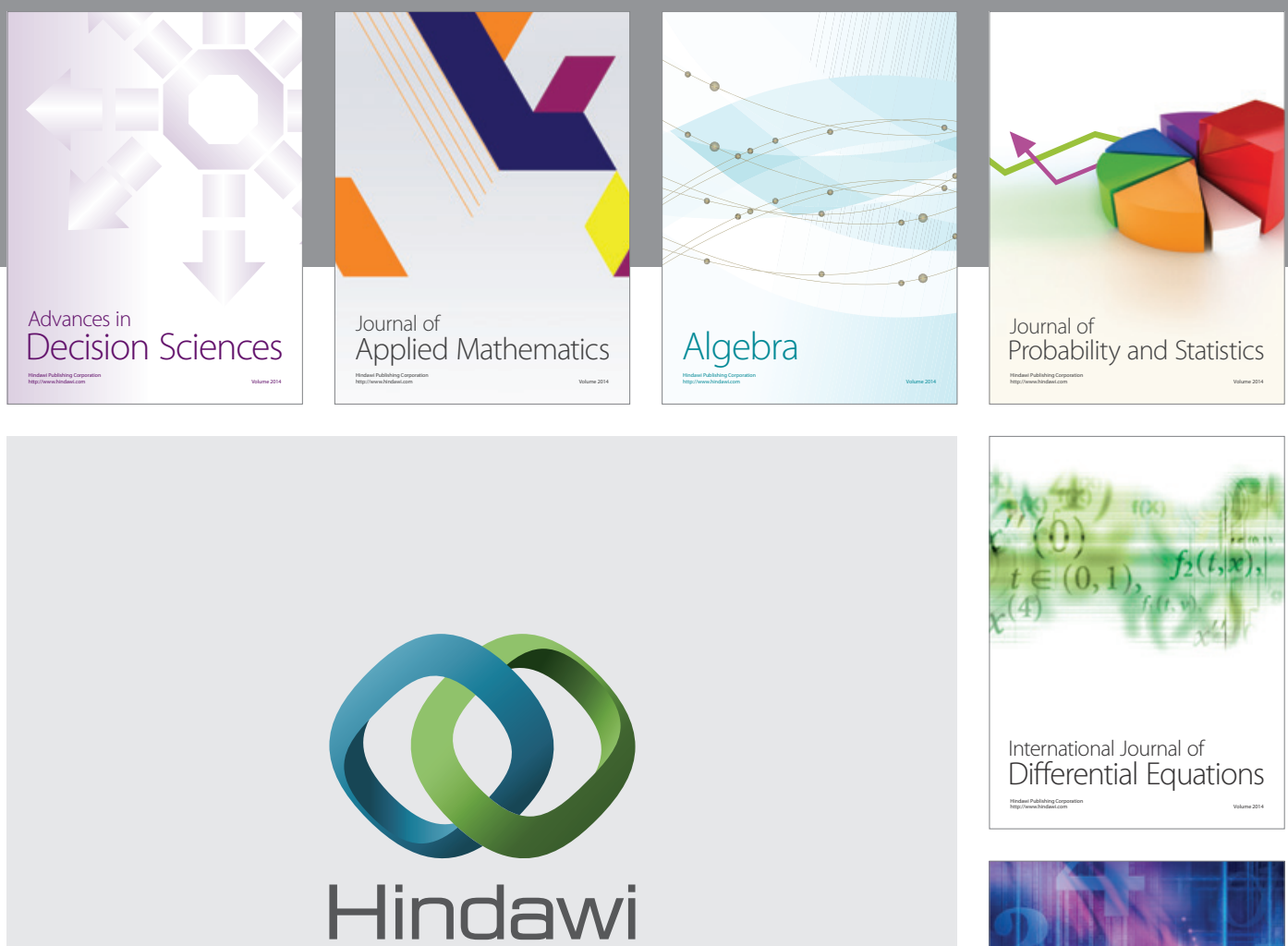

Submit your manuscripts at http://www.hindawi.com
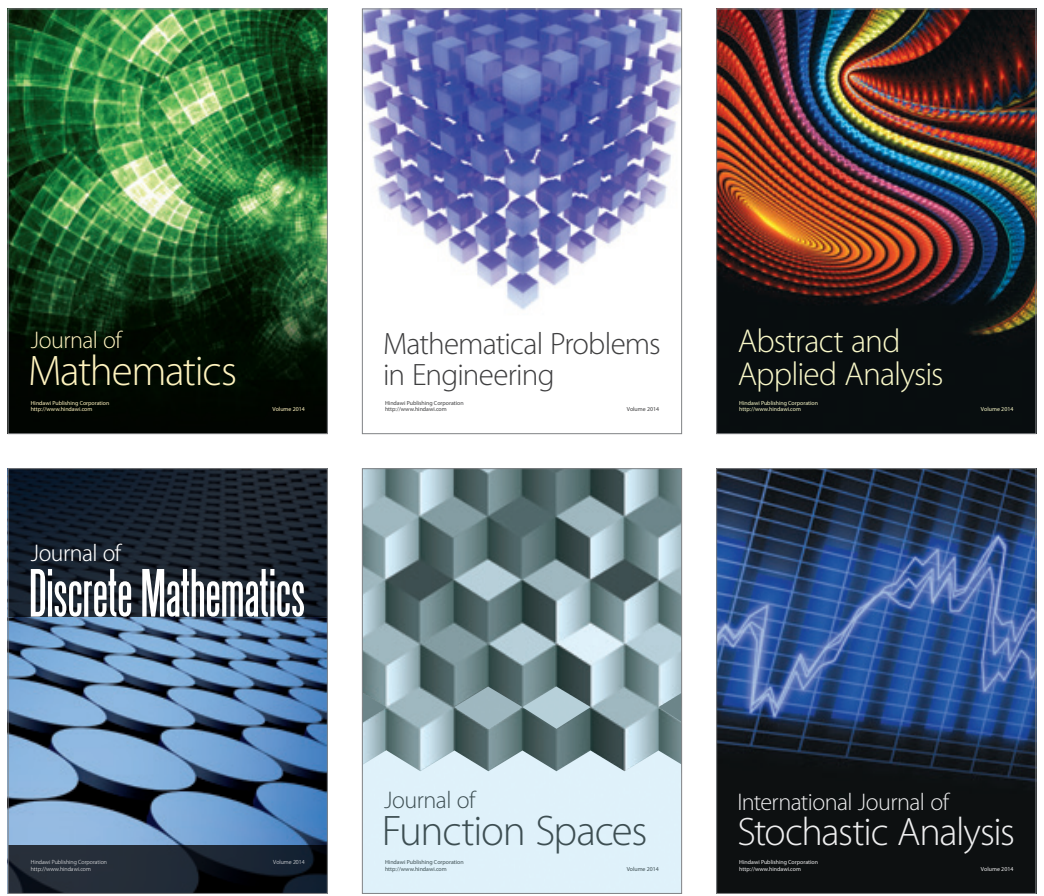

Journal of

Function Spaces

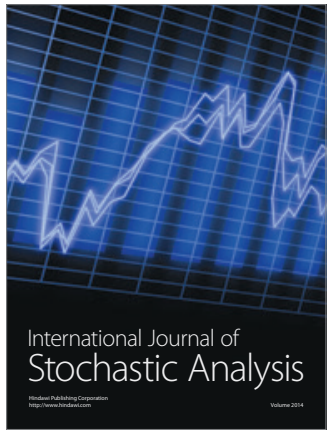

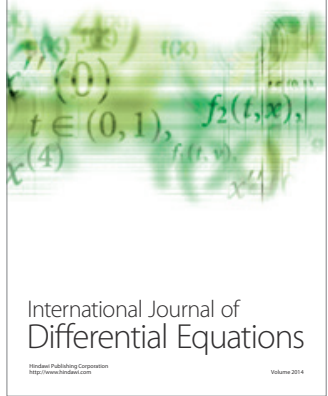
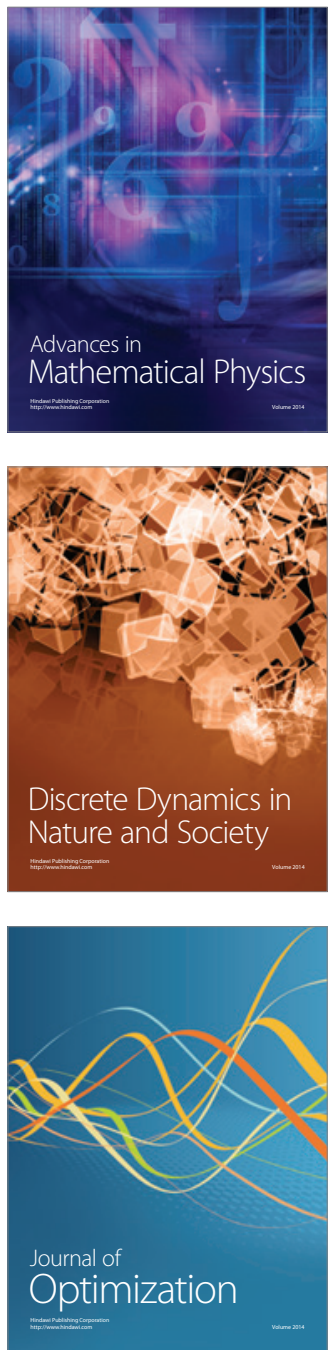